\title{
Morpho-physiological Response to Post-flowering Drought Stress in Small Red Seeded Common Bean (Phaseolus vulgaris L.) Genotypes
}

\author{
Yayis Rezene ${ }^{1}$, Setegn Gebeyehu ${ }^{2} \&$ Habtamu Zelleke ${ }^{3}$ \\ ${ }^{1}$ Areka Agricultural Research Center, P.O. Box 79, Areka, Ethiopia \\ ${ }^{2}$ Melkassa Agricultural Research Center, P.O. Box 436, Adama, Ethiopia \\ ${ }^{3}$ Haramaya University, P.O. Box 138, Dire Dawa, Ethiopia \\ Correspondence: Yayis Rezene, Areka Agricultural Research Center, P.O. Box 79, Areka, Ethiopia. E-mail: \\ rezene77@gmail.com
}

Received: October 15, 2012 Accepted: November 19, 2012 Online Published: November 30, 2012

doi:10.5539/jps.v2n1p42

URL: http://dx.doi.org/10.5539/jps.v2n1p42

\begin{abstract}
Common bean (Phaseolus vulgaris L.) is grown in regions where water deficit during reproductive development significantly reduces yield. The objective of this study was to assess morpho-physiological response to post-flowering drought stress in small red seeded common bean genotypes of diverse origin under field conditions. Forty-nine genotypes were evaluated in a $7 \times 7$ simple lattice design under two soil moisture regimes, non-stress (NS) and drought stress (DS) growth conditions in Gofa, Southern Ethiopia. Drought stress was initiated at flowering stage by withholding application of irrigation water. Significant differences were found among genotypes and between the soil moisture regimes except for days to flowering for the morpho-physiological traits considered. Drought stress reduced seed yield, harvest index (HI), seed per pod, seed per plant and 100-seed weight by 59\%,39\%, 15\%,29\% and 19\%, respectively. Drought-induced reduction in seed yield of the tested genotypes ranged from $9 \%$ in ECAB- 0427 to $89 \%$ in REDWOLAITA. With the highest yield of $1365.7 \mathrm{~kg}$ ha- 1 under drought stress, ECAB-0427 was the most drought tolerant genotypes, whereas with the lowest yield of $111.9 \mathrm{~kg}$ ha-1, REDWOLAITA was the most sensitive genotype to the stress imposed. Superior performance of ECAB-0427 under drought stress was attributed to the maintenance of higher leaf area index (LAI) (2.8) and pod harvest index (PHI) (67.6) compared with a LAI and PHI of 0.8 and 22.1, respectively, for REDWOLAITA. Yield under drought stress was correlated with yield under non-stress $(\mathrm{r}=0.68, p<0.01)$ implying that selection under drought conditions may lead to the identification of genotypes suitable for optimal conditions. Significantly higher correlation detected between GM (geometric mean) and seed yield under drought stress $(\mathrm{r}=0.95, p<0.01)$ entail that the drought index can be used as one of the most important selection criteria in identifying drought resistant small red bean genotypes.
\end{abstract}

Keywords: drought stress, non-stress, drought susceptibility index, geometric mean

\section{Introduction}

Common bean (Phaseolus vulgaris L.) is one of the principal grain legumes of eastern and southern Africa, occupying more than 4 million hectare annually and providing food for more than 100 million people (Wortmann et al., 1998). It is the second most important source of dietary protein and the third most important source of calories for lower income African households after cassava and maize (Broughton et al., 2003).

The National total area allocated for bean production in Ethiopia is currently estimated at 267.069 ha of land with a total production of about 0.33 million tons (CSA, 2009). The National and the regional (SNNPR) average yield of the crop is estimated to be 1.2 and 0.9 t/ha respectively (CSA, 2009). This is far lower than yield recorded at research sites using improved varieties (2.5-3 t/ha) (Asrat et al., 2006). Among the major production constraints drought stress is a serious agronomic problem and the single greatest abiotic factor contributing to common bean yield loss in southern Ethiopia (Asrat Asfaw, personal communication).

A better understanding of both the morphological, physiological and biochemical mechanisms involved in plant response to water deficit could therefore help to improve common bean productivity in dry land areas. Different mechanisms may make a drought tolerant plant. It may be by drought avoidance or drought tolerance (Blum \& Ebercon, 1981). Drought avoidance is the ability of a plant to escape periods of drought, particularly during the 
most sensitive periods of its development (Visser, 1994). Drought tolerance is the ability of the plant to endure or withstand a dry period by maintaining a favorable internal water balance under drought conditions. Loss of leaf area, which could result from reduced size of younger leaves and inhibition of the expansion of developing foliage, is also considered an adaptation mechanism to drought (Acosta-Gallegos, 1988). Early phenology coupled with rapid ground cover and dry matter production in legumes allows greater post-flower water-use leading to greater partitioning of dry matter into seeds (Siddique et al., 2001). Cultivars that show greater phenological adjustment exhibit higher seed yields under drought conditions (Acosta-Gallegos \& White, 1995). Significant differences have been observed for shoot biomass accumulation among common bean cultivars grown under moderate to severe drought stress conditions (Ramirez-Vallejo \& Kelly, 1998; Rosales-Serna et al., 2004)

According to Chaves et al. (2002), in addition to dry matter accumulation, the ability of genotypes to partition stored vegetative biomass to reproductive organs to a large extent determines sink establishment and economic yield under drought stress. Seed yield-based genotypic differences for drought resistance have been reported for common bean (Abebe et al., 1998; Teran \& Singh, 2002). Bean seed yield reduction due to drought stress are attributed to adverse effects of drought stress on individual yield components (number of pods per plant, number of seeds per pod, seed weight and harvest index). The relative importance of individual components as determinants of seed yield varies from experiment to experiment (Ramirez-Vallejo \& Kelly, 1998; Shenkut \& Brick, 2003). According to Rao et al. (2009) and Beebe et al. (2009) in addition to shoot biomass accumulation, leaf area index (LAI) and pod harvest index (PHI) have strong positive correlation with seed yield under drought conditions. Pod harvest index is considered as one of the key partitioning indices that measure the remobilization of photosynthates to seed. According to Beebe et al. (2009), PHI reflects plant efficiency in partition of photosynthates from vegetative shoot structures to pods and from pod wall to grain, which varies with the genotypes and is affected by drought. Strong positive associations have been reported between PHI and grain yield under drought stress and non-stress conditions (Polani et al., 2008; Beebe et al., 2008; Rao et al., 2009). In addition to yield components and plant attributes, the efficiency of partition of photosynthates to grain yield has also been the reliable approach to identify drought resistant genotypes under moisture stress conditions (Beebe et al., 2008; Rao et al., 2009).

The yields of common bean in the Goffa, southern Ethiopia region are lower than the national average due to frequent drought in the growing season and the drought-prone sandy-to-silt loam soils in the region. Drought during flowering and seed filling stages of the common bean, significantly limits crop productivity in this area. Therefore, field experiments were conducted to study the morphological and physiological responses of common bean genotypes to post flowering drought stress and indentify genotypes that are drought resistance.

\section{Material and Methods}

\subsection{Description of the Study Area}

The experiment was carried out during 2009 cropping season at Gofa, southern Ethiopia. The experimental site is located at 1348 masl and lies at $06^{\circ} 18^{\prime} 17.6^{\prime \prime} \mathrm{N}$ latitude and $036^{\circ} 50^{\prime} 28.5^{\prime \prime} \mathrm{E}$ longitude. The area with sandy loam soil receives an annual average rainfall of $758.5 \mathrm{~mm}$ with average maximum and minimum temperatures of 29.3 and $17.2{ }^{\circ} \mathrm{C}$, respectively.

\subsection{Plant Materials}

Forty-nine small red seeded common bean genotypes, constituted of inbred lines and released varieties obtained from local and international sources were used for the study. The inbred lines represent SER and SEA families, developed for specific adaptation to drought stress by CIAT, ECAB lines developed for East and Central African Bean growing environments and introduced by the National Bean Research Program and CAW and SNNP lines generated through crossing made at Awassa Agricultural Research Center. Five of the test genotypes were varieties released for wider or specific adaptation and a popular local variety cultivated in the area was also included.

\subsection{Experimental Design and Treatments}

The forty-nine genotypes were evaluated in $7 \times 7$ simple lattice design. Each genotype was planted on a plot made of two rows of $4 \mathrm{~m}$ length with a row-to-row distance of $0.6 \mathrm{~m}$ and a plant-to-plant spacing of $0.10 \mathrm{~m}$. The genotypes were grown under two soil moisture regimes, non-stress (NS) and drought stress (DS) conditions. Both watering regimes were managed uniformly following research recommendations. The total rain fall during the crop development period was $280 \mathrm{~mm}$. The soil moisture at planting was $83 \%$ at field capacity. For drought stressed treatments, drought was initiated at flowering by withholding application of irrigation, depending on the 
rain fall gravity irrigation at field capacity were applied every 10 to 12 days from planting until physiological maturity in the non-stress.

Water balance for each application was calculated based on excess or deficit water in the bean root zone relative to field capacity. Since the experiment was conducted during the dry season rain fall was minimal in the experimental period. Soil moisture measurements made at depths of 0-30, 31-60 and 61-90 $\mathrm{cm}$ at 10 days interval between planting and physiological maturity. Irrigation was applied when the root zone water deficit equaled the maximum allowable depletion of the available soil water. The soil moisture was monitored gravimetrically by oven drying the soil sample at $105^{\circ} \mathrm{C}$ for 24 hours to obtain the dry weight for each sampled plot. The soil moisture content was calculated as percent by weight using the formula, [(Wet weight $\mathrm{g}$ - dry weight g)/dry weight g)] x 100. For drought treatment a total of 3 irrigations were applied (each about $40.5 \mathrm{~mm}$ ) and supplemental irrigation was suspended after $80 \%$ of each plot flowered until the crop was physiologically mature. But the control experiment was kept irrigated until physiological maturity, and a total of 6 irrigations were given (each $40.5 \mathrm{~mm}$ ).

\subsection{Data Collection}

Days to flowering and days to maturity were recorded as the number of days from planting to when $50 \%$ of plants in a plot had at least one open flower and when $75 \%$ of plants in a plot had at least $90 \%$ of their pods dried, respectively. Leaf Area Index (LAI) was calculated as the ratio of total leaf area to ground area occupied by the plant. Total leaf area were determined by measuring the area of three leaves from top, middle and lower part of three randomly taken plants from each plots using CID-202 leaf area meter (CID, Inc., USA). Pod harvest index (PHI, \%), pod wall biomass proportion (PBP, \%) and pod partitioning index (PPI) were generated following drought phenotypic protocol (CIAT, unpublished). Seed yield and yield components (number of pods per plant, number of seeds per pod and 100-seed weight) were determined as described in CIAT (1995). Harvest index (HI) was calculated as the ratio of seed yield to total above-ground biomass multiplied by hundred. Geometric mean (GM) and drought sensitivity index (DSI) were calculated following the methods employed by Fernandez (1993) and Fisher and Maurer (1978), respectively. Percent reduction (PR) was calculated as [(mean value non-stress traits) - (mean value of drought stress trait)]/mean value of non-stress.

\subsection{Data Analysis}

The SAS computer package (SAS Institute, 2001) was used to test normality of residuals. Mean values were determined for all characters and these mean values were used in analysis of variance (ANOVA) to test genotype differences and the significance of genotypes x environment (NS \& DS) interaction effects. After testing the homogeneity of the error variance of the individual locations, combined analyses of variance over the two environments was performed as per the formula given by Gomez and Gomez (1984).

\section{Results}

Analysis of variance revealed high significant differences $(p<0.1)$ among the genotypes for all characters in the study except for days to flowering (DF) under both moisture regime. This indicates that there was adequate variability among the genotypes for days to maturity, days to seed filling, 100-seed wight, pods per plant, harvest indx, pod harvest index, pod wall biomass proportion, pod patition index leaf chlorophyll and canopy tempreture. However, pooled differences among the genotypes were non-significant for pods per plant, canopy temperature and leaf chlorophyll (Table 1). 
Table 1. Mean squares of eleven traits in 49 small red seeded common bean genotypes grown under stressed and non-stressed conditions

\begin{tabular}{llllll}
\hline Traits & WR & G & G X WR & Error & CV \\
d.f & 1 & 48 & 48 & 96 & \\
\hline DF & $20.89 \mathrm{~ns}$ & $16.40^{* *}$ & $7.34 * *$ & 2.33 & 3.7 \\
DM & $1681.00^{* *}$ & $74.92^{* *}$ & $13.32^{*}$ & 8.52 & 3.8 \\
DTSF & $2076.76^{* *}$ & $40.59^{* *}$ & $20.08^{* *}$ & 10.19 & 8.9 \\
HSW & $811.03^{* *}$ & $47.85^{* *}$ & $4.24 *$ & 2.65 & 8.9 \\
PPP & $1895.98^{* *}$ & $31.00^{* *}$ & $12.00 \mathrm{~ns}$ & 17.25 & 18.3 \\
HI & $1937.26^{* *}$ & $147.82^{* *}$ & $37.25^{* *}$ & 13.18 & 21.9 \\
PHI & $2005.12^{* *}$ & $642.74 * *$ & $103.95^{* *}$ & 49.23 & 21.9 \\
PBP & $3195.48^{* *}$ & $135.53 *$ & $157.92^{*}$ & 78.61 & 24.7 \\
PPI & $26606.27 * *$ & $745.48^{* *}$ & $621.32^{* *}$ & 223.75 & 27.0 \\
SPAD & $1999.37 * *$ & $28.32 *$ & $18.43 \mathrm{~ns}$ & 18.16 & 17.4 \\
CANT & $1830.62 * *$ & $3.01 \mathrm{~ns}$ & $3.57 \mathrm{~ns}$ & 3.85 & 9.3 \\
\hline
\end{tabular}

d.f=degree freedom, $\mathrm{WR}=$ watering regime; $\mathrm{G}=$ genotype $*$ =significant, $* *=$ highly significant, $\mathrm{ns}=$ non-significant at $p<0.05$ and 0.01 , respectively. $\mathrm{DF}=$ days to flowering; $\mathrm{DM}$ days to maturity, $\mathrm{DTSF}=$ days to seed filling, HSW=hundred seed weight (g), PPP=pods per plant, HI=harvest index $(\%)$, PHI pod harvest index $(\%), \mathrm{PBP}=$ pod wall biomass proportion (\%), PPI=pod partition index (\%), SPAD=leaf chlorophyll(SPAD), $\mathrm{CANT}=$ canopy temperature $\left({ }^{\circ} \mathrm{C}\right)$.

\subsection{Effect of Drought Stress on Morphological and Physiological Traits}

Significant genotypic differences were observed in LAI, PHI and PBP under drought stress growth conditions. Drought stress during the post flowering stage affected different morphological and physiological traits of small red seeded common bean genotypes (Table 2). Under drought stress condition genotypes MN-12643-1, SER-16, ICTAJU-95-14 and SER-125 were found to have with the highest mean values (3.3, 3.2, 3.2 and 3.1) of LAI respectively. However, CAW-02-01-1-1-3, REDWOLAITA and ECAB-0412 were found to have with lowest $(0.9,0.8$ and 0.8$)$ values of LAI respectively. Under similar growth conditions the genotypes ECAB-0427, SER-125, SER-109 and SER-16 exhibited the highest pod harvest index $(67.6,54.8,53.2$ and $47.6 \%$ respectively). Genotypes ECAB-0427, CAW-02-04-11-2-4 and SER-16 showed lowest values $(26.5,25.9$ and $25.5 \%$ respectively) of pod wall biomass proportion. The PHI values of $7.1 \%$ for ECAB- 0410 and $8.8 \%$ for CAW-02-03-8-11 with seed yields of 290.1 and $381.9 \mathrm{~kg} / \mathrm{ha}$ respectively were markedly lower than that of small red seeded common bean genotypes under drought stress condition (Table 3 ).

Table 2. Selected morphological and physiological traits of 49 small red seeded common bean genotypes grown under drought stress (DS) and non-stress (NS) growth conditions

\begin{tabular}{|c|c|c|c|c|c|c|c|c|c|c|}
\hline \multirow{2}{*}{ Genotype } & \multicolumn{2}{|c|}{ LAI } & \multicolumn{2}{|l|}{ PHI } & \multicolumn{2}{|l|}{ PBP } & \multicolumn{2}{|c|}{ SPAD } & \multicolumn{2}{|c|}{ CANT } \\
\hline & $\mathrm{DS}$ & NS & DS & NS & DS & NS & DS & NS & $\mathrm{DS}$ & NS \\
\hline ARS-R-93002 & 2.5 & 3 & 44 & 49.4 & 38.5 & 23.5 & 25.1 & 26.8 & 25.2 & 19 \\
\hline DOR-740 & 2.2 & 2.5 & 22.1 & 23 & 28.9 & 30.3 & 21 & 24.1 & 25.9 & 19.4 \\
\hline 790 RAA-34 & 1.1 & 4.4 & 17.2 & 22.5 & 41.8 & 38.2 & 22.3 & 27.1 & 24.6 & 20.8 \\
\hline ECAB-0427 & 2.8 & 3.5 & 67.6 & 60.2 & 26.5 & 25.2 & 22.2 & 34.9 & 25.5 & 17.3 \\
\hline ECAB-0424 & 1.6 & 3.7 & 16.4 & 8.4 & 40.8 & 30.5 & 18.7 & 20.5 & 22.5 & 17.7 \\
\hline ECAB-0410 & 1.9 & 3.4 & 7.1 & 6.4 & 33.1 & 29.3 & 25.7 & 27.6 & 22.8 & 18.3 \\
\hline MN-12643-1 & 3.3 & 3.9 & 13.1 & 21.8 & 30.4 & 24.2 & 26 & 33 & 22.2 & 17.7 \\
\hline ECAB-0412 & 0.8 & 4.4 & 18.7 & 32.4 & 41.8 & 25.7 & 17.9 & 19 & 24.2 & 18.1 \\
\hline ICTAJU-95-14 & 3.2 & 3.3 & 45.6 & 51.1 & 33.6 & 32.6 & 22.2 & 30 & 24.7 & 17.3 \\
\hline
\end{tabular}




\begin{tabular}{|c|c|c|c|c|c|c|c|c|c|c|}
\hline LR-93201338 & 1.3 & 3.3 & 30.2 & 5 & 32.8 & 24.9 & 24.5 & 24.8 & 25.1 & 17.1 \\
\hline DOR-721 & 2 & 2.6 & 21.3 & 16.7 & 50.1 & 29.5 & 21.6 & 32.5 & 25.1 & 18.2 \\
\hline T842 6F 12-3 & 2 & 3 & 24.3 & 12.3 & 34.4 & 30.4 & 25.2 & 33.5 & 24.7 & 18.6 \\
\hline RCB-592 & 2.5 & 3.3 & 29.8 & 51.6 & 31.4 & 27.5 & 21.8 & 30.1 & 23.4 & 19.2 \\
\hline SER-48 & 2.8 & 3.5 & 39.8 & 54.9 & 34.8 & 27.6 & 16.9 & 27.8 & 24 & 16.7 \\
\hline SER-78 & 2.4 & 2.7 & 41.6 & 55.2 & 42.2 & 27.7 & 18.7 & 28.1 & 23.6 & 16.5 \\
\hline SER-95 & 2.1 & 2.4 & 33.8 & 35.5 & 51.1 & 29.6 & 18 & 29.1 & 23.6 & 17.3 \\
\hline SER-118 & 3 & 3.3 & 37.9 & 50.4 & 30.4 & 28.4 & 17.5 & 28.3 & 23.2 & 19.1 \\
\hline SER-119 & 2.2 & 3.2 & 40 & 41.2 & 36.5 & 30.9 & 22.8 & 32.1 & 27.3 & 17.6 \\
\hline SER-125 & 3.1 & 3.1 & 54.8 & 56.3 & 34.9 & 33.8 & 22.2 & 26.1 & 24.7 & 18.8 \\
\hline SER-128 & 2.1 & 3.6 & 43.1 & 56.1 & 42 & 37.9 & 21.2 & 28.7 & 25.5 & 17.3 \\
\hline SER-176 & 1.5 & 2.5 & 34.1 & 34.8 & 54 & 25.2 & 21.9 & 27.1 & 25.1 & 18.3 \\
\hline SER-180 & 2.1 & 3.1 & 36.5 & 40.5 & 35 & 33.7 & 23.5 & 32.9 & 27.5 & 18.4 \\
\hline SER-194 & 2 & 3.3 & 45.3 & 60.2 & 48.7 & 38.3 & 21.8 & 25.7 & 26.3 & 17.5 \\
\hline NASIER & 3 & 3.5 & 21.3 & 42.8 & 40.4 & 26.4 & 19.2 & 27.2 & 25.5 & 17.7 \\
\hline DINKNESH & 2.1 & 3.7 & 12.2 & 22.4 & 46.6 & 24.6 & 19.7 & 23.9 & 25 & 17.7 \\
\hline CAW-02-03-8-11 & 2 & 3.5 & 8.8 & 18.1 & 48.1 & 25.4 & 23.8 & 25.7 & 26.4 & 19.5 \\
\hline CAW-02-05-2-7-5 & 2.5 & 3.6 & 20.3 & 34.6 & 38.6 & 31.6 & 15.6 & 31.2 & 24.2 & 17.9 \\
\hline CAW-02-04-7-6-7 & 2.1 & 3.2 & 30.7 & 25.2 & 50.4 & 25.5 & 20.2 & 29.8 & 23.1 & 17.5 \\
\hline SNNPR1-35 & 2.8 & 3.4 & 22.5 & 20.6 & 30.9 & 28 & 23.2 & 27 & 24.2 & 16.7 \\
\hline CAW-02-04-11-2-4 & 2.7 & 2.9 & 39.7 & 43.6 & 25.9 & 67.4 & 17.3 & 24.3 & 25.6 & 15.7 \\
\hline CAW-02-03-1-6-44 & 2.7 & 3.6 & 22.1 & 38 & 45.8 & 26 & 18.1 & 27.3 & 23.3 & 17.2 \\
\hline CAW-02-04-4-11-4 & 1.9 & 3.7 & 41.5 & 36.8 & 35.2 & 23.6 & 19.5 & 30.6 & 27.3 & 18.1 \\
\hline SER-43 & 1.3 & 2.5 & 31.3 & 50.6 & 33.2 & 34.7 & 21 & 26.3 & 23.6 & 18.7 \\
\hline SER-16 & 3.2 & 3.7 & 47.6 & 44.1 & 25.5 & 32.8 & 22.9 & 23.9 & 23.3 & 17.7 \\
\hline CAW-02-04-8-3-1 & 2.8 & 3.8 & 23 & 43 & 47.8 & 45.3 & 23.7 & 29 & 21.2 & 18.9 \\
\hline SEA-5 & 3 & 3.1 & 25.8 & 37.5 & 41.2 & 25.4 & 26.2 & 34.1 & 21.2 & 18.6 \\
\hline VAX-6 & 2.5 & 3 & 28.1 & 22.1 & 45 & 50.9 & 18.1 & 23.8 & 22.2 & 19 \\
\hline OMO-95 & 1.6 & 3.1 & 13.1 & 29.1 & 53.2 & 24.2 & 23.7 & 26.9 & 22.5 & 17.9 \\
\hline LR-93201347 & 3 & 3.6 & 21.2 & 30.4 & 31.7 & 31.6 & 22.2 & 18.7 & 24.4 & 18.7 \\
\hline CAW-02-01-1-1-3 & 2.4 & 2.8 & 20.5 & 35 & 36.8 & 27 & 20.2 & 32.4 & 24.3 & 18.1 \\
\hline SER-109 & 2.2 & 3.8 & 53.2 & 48.8 & 34.2 & 38.2 & 20.3 & 19.9 & 24.3 & 17.8 \\
\hline CAW-02-01-5-1-2 & 0.9 & 2.7 & 13.6 & 23.8 & 42.9 & 49.4 & 25 & 25.8 & 23.2 & 18.4 \\
\hline ECAB-0416 & 1.9 & 3.2 & 23.5 & 31.4 & 36.9 & 27.4 & 20.5 & 30.7 & 25.5 & 17 \\
\hline SER-178 & 1.6 & 4.2 & 30.4 & 34.8 & 60.3 & 24.8 & 21.7 & 28.2 & 23.1 & 19.2 \\
\hline K 26/35 CF 10-9 & 1.6 & 3.8 & 13 & 37.6 & 41.2 & 44.9 & 19.1 & 35.7 & 25.1 & 18.1 \\
\hline CAW-02-01-1-1-1 & 1.3 & 2 & 15.2 & 40.2 & 53.8 & 30.1 & 22.2 & 31.8 & 26.2 & 20.2 \\
\hline RED WOLITA & 0.8 & 4.3 & 22.1 & 28.5 & 50.7 & 28.9 & 18.9 & 23.2 & 23 & 19.4 \\
\hline HAWASSA DUME & 2 & 3.6 & 28.7 & 32.8 & 50.2 & 49.4 & 22.5 & 24.2 & 23.2 & 20.4 \\
\hline LAOCALVARIETY & 2.4 & 3.6 & 18.6 & 27.7 & 35.6 & 31.5 & 18.3 & 23.5 & 22.2 & 18.3 \\
\hline MEANS & 2.1 & 3.3 & 28.8 & 35.2 & 39.9 & 31.8 & 21.2 & 27.6 & 24.3 & 18.1 \\
\hline $\mathrm{LSD}_{0.05}$ & 0.9 & ns & 12.6 & 15.4 & 13.8 & ns & ns & ns & ns & ns \\
\hline $\mathrm{CV}$ & 21.8 & 27.9 & 21.8 & 21.9 & 17.3 & 32.9 & 16.2 & 17.9 & 8.5 & 10.3 \\
\hline
\end{tabular}

$\mathrm{LAI}=$ leaf area index, $\mathrm{PHI}=$ pod harvest index, $\mathrm{PBP}=$ pod wall biomass proportion, $\mathrm{SPAD}=$ leaf chlorophyll, $\mathrm{CANT}=$ canopy temperature $\left({ }^{\circ} \mathrm{C}\right)$. 
Table 3. Seed yield under non-stress (NS), drought stress (DS) and seed yield based geometric mean (GM), percent reduction (PR) and drought susceptibility index (DSI) for 49 small red common bean genotypes grown under non-stress (NS) and drought stress (DS) conditions

\begin{tabular}{|c|c|c|c|c|c|}
\hline \multirow[b]{2}{*}{ Genotype } & \multicolumn{3}{|c|}{ Seed yield $\left(\mathrm{kg} \mathrm{ha}^{-1}\right)$} & \multirow[b]{2}{*}{ GM } & \multirow[b]{2}{*}{ DSI } \\
\hline & NS & DS & PR & & \\
\hline ARS-R-93002 & 1830.7 & 890.5 & 0.51 & 1276.7 & 0.85 \\
\hline DOR-740 & 711.4 & 420.5 & 0.42 & 546.9 & 0.7 \\
\hline 790 RAA-34 & 288.9 & 129.9 & 0.55 & 193 & 0.9 \\
\hline ECAB-0427 & 1765.6 & 1365.7 & 0.22 & 1548.8 & 0.35 \\
\hline ECAB-0424 & 1192.2 & 262.2 & 0.76 & 551 & 1.35 \\
\hline ECAB-0410 & 982 & 290.1 & 0.71 & 532.6 & 1.15 \\
\hline MN-12643-1 & 2170 & 958.2 & 0.56 & 1441.8 & 0.9 \\
\hline ECAB-0412 & 993.1 & 114.1 & 0.89 & 335.8 & 1.35 \\
\hline ICTAJU-95-14 & 2144.5 & 1260.9 & 0.42 & 1643.6 & 0.55 \\
\hline LR-93201338 & 693.4 & 198.8 & 0.72 & 363.2 & 1.2 \\
\hline DOR-721 & 537.7 & 500.1 & 0.09 & 518.4 & 0.15 \\
\hline $\mathrm{T} 842$ 6F 12-3 & 875 & 412.7 & 0.51 & 597.6 & 0.9 \\
\hline RCB-592 & 3002.6 & 975 & 0.68 & 1698.5 & 1.3 \\
\hline SER-48 & 1437.9 & 1220.8 & 0.17 & 1323.7 & 0.25 \\
\hline SER-78 & 1901 & 660.6 & 0.66 & 1116.8 & 1.1 \\
\hline SER-95 & 1559 & 457.6 & 0.69 & 830.2 & 1.15 \\
\hline SER-118 & 1753.8 & 730.6 & 0.58 & 1127.6 & 0.95 \\
\hline SER-119 & 2082.5 & 808.5 & 0.6 & 1287.6 & 0.95 \\
\hline SER-125 & 2015.9 & 1183 & 0.41 & 1542.8 & 0.45 \\
\hline SER-128 & 1873.7 & 828.2 & 0.55 & 1243.7 & 0.9 \\
\hline SER-176 & 621.3 & 278.6 & 0.52 & 415 & 0.9 \\
\hline SER-180 & 894.2 & 500.7 & 0.44 & 669 & 0.75 \\
\hline SER-194 & 2632.8 & 999.3 & 0.62 & 1615.9 & 1 \\
\hline NASIER & 2029.7 & 908.2 & 0.54 & 1350.4 & 0.85 \\
\hline DINKNESH & 1467.5 & 456.7 & 0.68 & 816.8 & 1.1 \\
\hline CAW-02-03-8-11 & 1619.7 & 381.9 & 0.77 & 786.3 & 1.25 \\
\hline CAW-02-05-2-7-5 & 1945 & 569.1 & 0.7 & 1046.4 & 1.2 \\
\hline CAW-02-04-7-6-7 & 1868.2 & 581.4 & 0.69 & 1042.2 & 1.1 \\
\hline SNNPR1-35 & 1522.9 & 727.6 & 0.42 & 984.6 & 0.7 \\
\hline CAW-02-04-11-2-4 & 2264.9 & 773.6 & 0.66 & 1319.7 & 1.1 \\
\hline CAW-02-03-1-6-44 & 1701.8 & 545.5 & 0.68 & 963.3 & 1.1 \\
\hline CAW-02-04-4-11-4 & 1241.8 & 420.2 & 0.69 & 720.7 & 1.25 \\
\hline SER-43 & 1810 & 650.1 & 0.63 & 1079.8 & 1.05 \\
\hline SER-16 & 2791.8 & 1225.1 & 0.56 & 1849.4 & 0.78 \\
\hline CAW-02-04-8-3-1 & 1666.6 & 857.1 & 0.44 & 1183.3 & 0.75 \\
\hline SEA-5 & 1152 & 595.4 & 0.5 & 813.5 & 0.8 \\
\hline VAX-6 & 720.3 & 412.4 & 0.35 & 531.3 & 0.55 \\
\hline
\end{tabular}




\begin{tabular}{llllll}
\hline OMO-95 & 1255.3 & 251.3 & 0.8 & 535.7 & 1.4 \\
LR-93201347 & 918.3 & 304.3 & 0.67 & 528.3 & 1.1 \\
CAW-02-01-1-1-3 & 944.5 & 165 & 0.69 & 362.9 & 0.95 \\
SER-109 & 2131.6 & 891.5 & 0.59 & 1375.5 & 1 \\
CAW-02-01-5-1-2 & 552.4 & 131.2 & 0.69 & 253.8 & 1.1 \\
ECAB-0416 & 1484.7 & 431.1 & 0.72 & 796.5 & 1.2 \\
SER-178 & 1931.4 & 258.7 & 0.87 & 703.1 & 1.45 \\
K 26/35 CF 10-9 & 1267.2 & 257.6 & 0.81 & 567.1 & 1.3 \\
CAW-02-01-1-1-1 & 649.8 & 198.9 & 0.69 & 349.3 & 1.15 \\
RED WOLITA & 948.8 & 112 & 0.89 & 325.1 & 1.45 \\
HAWASSA DUME & 2005.3 & 356.5 & 0.83 & 834.8 & 1.35 \\
LOCAL VARIETY & 1279.4 & 405.5 & 0.65 & 679.6 & 1.05 \\
MEAN & 1492 & 577.9 & 0.59 & 902.4 & 0.98 \\
LSD 0.05 & 696 & 373.8 & 0.56 & 0.3 & 3.05 \\
CV & 23.2 & 30.3 & 26.05 & 20.6 & 28.88 \\
\hline
\end{tabular}

\subsection{Effect of Drought Stress on Seed Yield and Yield Components}

Seed yield of all genotypes subjected to drought stress (DS) was significantly lower than the non-stressed (NS) counterparts (Table 3). Seed yield, pods per plant, 100-seed weight and harvest index of small red seeded common bean genotypes, were significantly reduced by drought stress. The largest reduction in seed yield due to drought stress was in genotypes REDWOLAITA (89\%) and ECAB $0412(89 \%)$ followed by SER-178 (87\%). These genotypes are sensitive to drought stress. In contrast SER-48, DOR-721 and ECAB-0427 showed lower yield reduction $17 \%, 0.09 \%$ and $22 \%$ respectively when exposed to drought stress. In a similar way 100 -seed weight of genotypes REDWOLAITA and ECAB-0412 were highly affected by drought stress (30\%). Twelve genotypes (ECAB-0424, ECAB-0410, ECAB-0416, ECAB-0412, REDWOLAITA, HAWASSADUME, CAW-02-03-8-11, CAW-02-05-2-7-5,CAW-02-04-4-11-4, RCB-592, SER-178, and K26/3510-9) showed higher values of DSI $(1.35,1.15,1.20,1.45,1.35,1.25,1.20,1.25,1.25,1.30,1.45$ and 1.30 respectively). DSI for seed yield were lowest (0.15 and 0.17) for genotypes DOR-721 and SER-48 respectively.

Seed yield of the genotypes under drought stress condition ranged from $112.0 \mathrm{~kg} / \mathrm{ha}$ for REDWOLAITA to $1365.70 \mathrm{~kg} / \mathrm{ha}$ for ECAB-0427. Seven of the genotypes ECAB-0427, ICTAJU-95-14, SER-16, SER-48, SER-125, SER-194 and RCB-592 with seed yields of $1365.70 \mathrm{~kg} / \mathrm{ha}, 1260.90 \mathrm{~kg} / \mathrm{ha}, 1225.52 \mathrm{~kg} / \mathrm{ha}, 1220.00$ $\mathrm{kg} / \mathrm{ha}, 1183.00 \mathrm{~kg} / \mathrm{ha}, 999.3 \mathrm{~kg} / \mathrm{ha}$ and $975.0 \mathrm{~kg} / \mathrm{ha}$ coupled with higher GM values 1548.8, 1643.6, 1849.4, $1323.7,1542.8,1615.9$, and 1698.5 respectively were outstanding in their adaptation to drought stress environment. Among the genotypes tested REDWOLAITA produced the lowest seed yield of $112.0 \mathrm{~kg} / \mathrm{ha}$ under drought stress condition (Table 3). On average drought stress during post flowering stage reduced seed yield by $59 \%$ (Table 3), 100 -seed weight by $19 \%$, seed per pod by $15 \%$, pods per plant by $29 \%$ and harvest index by $39 \%$ (Table 4).

Table 4. Hundred seed weight (HSW), seed per pod (SPP), pod per plant (PPP), harvest index(HI) and theirpercent reduction (PR) of 49 small red seeded common bean genotypes grown under drought stress (DS) and non-stress (NS) growth conditions at Gofa, southern Ethiopia

\begin{tabular}{llllllllllllll}
\hline & HSW & \multicolumn{4}{c}{ SPP } & \multicolumn{4}{c}{ PPP } & \multicolumn{1}{c}{ HI } \\
Genotype & NS & DS & PR & NS & DS & PR & NS & DS & PR & NS & DS & PR \\
\hline ARS-R-93002 & 26.5 & 24.7 & 0.07 & 5 & 4.6 & 0.08 & 23.9 & 13 & 0.46 & 30.1 & 15 & 0.5 \\
DOR-740 & 18.2 & 16.7 & 0.08 & 5.3 & 5 & 0.06 & 17.8 & 16.7 & 0.06 & 11.6 & 11.5 & 0.01 \\
790 RAA-34 & 15.5 & 14.9 & 0.04 & 3.9 & 2.7 & 0.31 & 15 & 12.6 & 0.16 & 15.6 & 8.1 & 0.48 \\
ECAB-0427 & 27.1 & 24.9 & 0.08 & 5.3 & 4.7 & 0.11 & 17.3 & 16 & 0.08 & 29.7 & 22.9 & 0.23 \\
\hline
\end{tabular}




\begin{tabular}{|c|c|c|c|c|c|c|c|c|c|c|c|c|}
\hline ECAB-0424 & 17.9 & 13.6 & 0.24 & 4.9 & 4.8 & 0.02 & 16.3 & 10.1 & 0.38 & 14.7 & 4.4 & 0.7 \\
\hline ECAB-0410 & 15.5 & 14 & 0.1 & 4.6 & 3.1 & 0.33 & 19.9 & 15.2 & 0.24 & 13 & 3.9 & 0.7 \\
\hline MN-12643-1 & 17.4 & 14.2 & 0.18 & 4.7 & 3.6 & 0.23 & 22.4 & 18 & 0.2 & 20 & 10.1 & 0.5 \\
\hline ECAB-0412 & 18.1 & 16.3 & 0.1 & 4 & 2.5 & 0.38 & 15.7 & 13.3 & 0.15 & 18.5 & 2.9 & 0.84 \\
\hline ICTAJU-95-14 & 33.5 & 27.1 & 0.19 & 4.7 & 4.4 & 0.06 & 19.4 & 18.3 & 0.06 & 26.2 & 23.3 & 0.11 \\
\hline LR-93201338 & 15.6 & 13.1 & 0.16 & 3 & 2.8 & 0.07 & 18.2 & 13.6 & 0.25 & 17.7 & 4.2 & 0.76 \\
\hline DOR-721 & 17.7 & 15.1 & 0.15 & 5.2 & 4.9 & 0.06 & 16.2 & 13.4 & 0.17 & 13.9 & 10.7 & 0.23 \\
\hline T842 6F 12-3 & 15.9 & 14.8 & 0.07 & 4.9 & 4.8 & 0.02 & 20.9 & 14 & 0.33 & 14.7 & 8 & 0.46 \\
\hline RCB-592 & 28.2 & 22.6 & 0.2 & 4.8 & 4.7 & 0.02 & 21.3 & 17.3 & 0.19 & 25.3 & 19.4 & 0.23 \\
\hline SER-48 & 27.2 & 20.5 & 0.25 & 4.4 & 4 & 0.09 & 23.8 & 17.2 & 0.28 & 30.1 & 19 & 0.37 \\
\hline SER-78 & 19.5 & 15.4 & 0.21 & 5 & 4.5 & 0.1 & 22.2 & 16.6 & 0.25 & 28.4 & 14.8 & 0.48 \\
\hline SER-95 & 21.2 & 17 & 0.2 & 4 & 3.6 & 0.1 & 20.2 & 16.4 & 0.19 & 23.2 & 11.3 & 0.51 \\
\hline SER-118 & 21.9 & 15.2 & 0.31 & 5.6 & 4.3 & 0.23 & 20.5 & 16.5 & 0.2 & 24.3 & 21 & 0.14 \\
\hline SER-119 & 24.3 & 16.7 & 0.31 & 4.6 & 4.3 & 0.07 & 19.4 & 14.3 & 0.26 & 28.2 & 22.3 & 0.21 \\
\hline SER-125 & 24.3 & 20.4 & 0.16 & 4.7 & 4.3 & 0.09 & 18 & 15.6 & 0.13 & 28 & 18.9 & 0.33 \\
\hline SER-128 & 26 & 19.1 & 0.27 & 4.6 & 3.5 & 0.24 & 23.8 & 18.2 & 0.24 & 22.8 & 16.3 & 0.29 \\
\hline SER-176 & 18.6 & 16.3 & 0.12 & 4.4 & 3.5 & 0.2 & 17.3 & 13.7 & 0.21 & 15 & 13.4 & 0.11 \\
\hline SER-180 & 19.6 & 14.7 & 0.25 & 3.8 & 3 & 0.21 & 23.9 & 17.8 & 0.26 & 15 & 9 & 0.4 \\
\hline SER-194 & 25.4 & 18.2 & 0.28 & 4.7 & 2.6 & 0.45 & 18.9 & 17.6 & 0.07 & 29.8 & 24.5 & 0.18 \\
\hline NASIER & 22.1 & 14.2 & 0.36 & 5.3 & 3.9 & 0.26 & 17.9 & 9.9 & 0.45 & 27.4 & 20.4 & 0.26 \\
\hline DINKNESH & 19.5 & 16.5 & 0.15 & 4.3 & 3.6 & 0.16 & 19.1 & 11.3 & 0.41 & 17.8 & 8.9 & 0.5 \\
\hline CAW-02-03-8-11 & 19.3 & 16.1 & 0.17 & 5.8 & 4.6 & 0.21 & 14.8 & 11 & 0.26 & 14.8 & 8.1 & 0.45 \\
\hline CAW-02-05-2-7-5 & 19.9 & 15.7 & 0.21 & 4.9 & 4.2 & 0.14 & 21.8 & 13 & 0.4 & 20.1 & 10.4 & 0.48 \\
\hline CAW-02-04-7-6-7 & 17.9 & 14.6 & 0.18 & 5.8 & 5 & 0.14 & 25.5 & 18.2 & 0.29 & 21 & 12.3 & 0.41 \\
\hline SNNPR1-35 & 20.3 & 17.8 & 0.12 & 4.2 & 3.6 & 0.14 & 22.3 & 17.8 & 0.2 & 21.6 & 17 & 0.21 \\
\hline CAW-02-04-11-2-4 & 20.7 & 16.1 & 0.22 & 5.3 & 3.7 & 0.3 & 22.3 & 15.2 & 0.32 & 21.8 & 16 & 0.27 \\
\hline CAW-02-03-1-6-44 & 18.5 & 14.8 & 0.2 & 4 & 3.8 & 0.05 & 22.9 & 12.4 & 0.46 & 16.4 & 10 & 0.39 \\
\hline CAW-02-04-4-11-4 & 23 & 17.1 & 0.26 & 5.3 & 3 & 0.43 & 20.7 & 15.3 & 0.26 & 15.7 & 12.8 & 0.18 \\
\hline SER-43 & 21.6 & 15.2 & 0.3 & 5 & 5 & 0 & 20.6 & 16.4 & 0.2 & 23.1 & 12.6 & 0.45 \\
\hline SER-16 & 24.5 & 15.6 & 0.36 & 5.3 & 5.3 & 0 & 29.3 & 18.1 & 0.38 & 31.3 & 31 & 0.01 \\
\hline CAW-02-04-8-3-1 & 18.9 & 14.5 & 0.23 & 5.2 & 4.1 & 0.21 & 29.6 & 16.8 & 0.43 & 18.2 & 14.5 & 0.2 \\
\hline SEA-5 & 24 & 22.1 & 0.08 & 4.8 & 3.3 & 0.31 & 23.8 & 15.7 & 0.34 & 17.4 & 14 & 0.2 \\
\hline VAX-6 & 16.1 & 15.8 & 0.02 & 4.6 & 4.6 & 0 & 20.7 & 13.3 & 0.36 & 13.9 & 11.5 & 0.17 \\
\hline OMO-95 & 17.4 & 13.6 & 0.22 & 4.7 & 4.6 & 0.02 & 17.2 & 11.1 & 0.35 & 28.1 & 17.9 & 0.36 \\
\hline LR-93201347 & 16.4 & 14.2 & 0.13 & 3.9 & 3.3 & 0.15 & 15.7 & 11.8 & 0.25 & 14 & 6.8 & 0.51 \\
\hline CAW-02-01-1-1-3 & 17.4 & 13.3 & 0.24 & 4.1 & 3.8 & 0.07 & 27.5 & 12.2 & 0.56 & 18.7 & 4.4 & 0.76 \\
\hline SER-109 & 22.8 & 17.9 & 0.21 & 4.5 & 4.2 & 0.07 & 25.9 & 17.2 & 0.34 & 28.7 & 16.9 & 0.41 \\
\hline CAW-02-01-5-1-2 & 18.4 & 13.9 & 0.24 & 5.1 & 4.7 & 0.08 & 23.8 & 8.7 & 0.63 & 9.1 & 3.5 & 0.62 \\
\hline ECAB-0416 & 18.4 & 14 & 0.24 & 4.9 & 4.2 & 0.14 & 17.3 & 13.6 & 0.21 & 16.7 & 9 & 0.46 \\
\hline SER-178 & 23.3 & 16.6 & 0.29 & 5 & 3.9 & 0.22 & 19.2 & 12.2 & 0.36 & 23.7 & 8.7 & 0.63 \\
\hline K 26/35 CF 10-9 & 18.9 & 13.9 & 0.26 & 5 & 4.8 & 0.04 & 26.2 & 13.7 & 0.48 & 17 & 6.6 & 0.61 \\
\hline CAW-02-01-1-1-1 & 17.6 & 14.3 & 0.19 & 4.1 & 3.1 & 0.24 & 18.8 & 11.3 & 0.4 & 11 & 5.2 & 0.53 \\
\hline
\end{tabular}




\begin{tabular}{lllllllllllll}
\hline RED WOLITA & 20.3 & 14.4 & 0.29 & 4.6 & 4.1 & 0.11 & 13.2 & 8.2 & 0.38 & 14.5 & 4.4 & 0.7 \\
HAWASSA DUME & 19.6 & 16.4 & 0.16 & 4.9 & 4.2 & 0.14 & 19.2 & 13.9 & 0.28 & 30.4 & 13.2 & 0.57 \\
LOCAL VARIETY & 14.5 & 12.6 & 0.13 & 4.8 & 4.2 & 0.13 & 20.9 & 10.2 & 0.51 & 16 & 13.4 & 0.16 \\
MEAN & 20.5 & 16.4 & 0.19 & 4.6 & 4.3 & 0.15 & 20.6 & 14.3 & 0.29 & 19.7 & 13.4 & 0.39 \\
LSD $_{0.05}$ & 3.1 & 3.5 & & NS & 1.2 & & 8.2 & 3.9 & & 7.7 & 6.8 & \\
CV & 7.4 & 10.5 & & 17.2 & 14.3 & & 19.8 & 13.8 & & 19.7 & 25.2 & \\
\hline
\end{tabular}

\subsection{Association among Trait}

Correlation coefficients between the NS and DS environments were positive and highly significant $(\mathrm{p}<0.01)$ for seed yield and 100-seed weight (Table 5). Seed yield in DS environments was negatively correlated with percent reduction (PR) and drought susceptible index (DSI). Seed yield under drought were positively correlated with seed yield under non-stress environment $\left(\mathrm{r}=0.68^{* *}\right)$ at drought stress level of $\mathrm{DII}=0.61$. There was a positive association between percent reduction (PR) and drought susceptibility index (DSI) for seed yield ( $\left.\mathrm{r}=0.97^{* *}\right)$.

Table 5. Correlations(r) among non stressed (NS), Drought stress (DS), Geometric mean (GM), percent reduction (PR), and drought susceptible index (DSI) for seed yield $(\mathrm{kg} / \mathrm{ha})$ and 100-seed weight (g) for 49 small red bean genotypes evaluated both in NS and DS at Gofa, southern Ethiopia in 2009

\begin{tabular}{llllllllll}
\hline & \multicolumn{3}{c}{ seed yield (Kg/ha) } & \multicolumn{5}{l}{100 -seed weight $(\mathrm{g})$} \\
\cline { 2 - 9 } & & DS & GM & PR & DSI & NS & DS & GM & PR \\
\hline Seed yield (kg/ha) & NS & $0.68^{* *}$ & $0.87^{* *}$ & 0.11 & 0.09 & $0.63^{* *}$ & $0.40^{* *}$ & $0.57^{* *}$ & $0.34^{* *}$ \\
& DS & & $0.95^{* *}$ & $-0.60^{* *}$ & $-0.59^{* *}$ & $0.62^{* *}$ & $0.56^{* *}$ & $0.63^{* *}$ & 0.1 \\
& GM & & & $-0.33^{* *}$ & $-0.32^{* *}$ & $0.69^{* *}$ & $0.54^{* *}$ & $0.66^{* *}$ & $0.23^{*}$ \\
& PR & & & & $0.97^{* *}$ & -0.16 & $-0.36^{* *}$ & $-0.25^{*}$ & $0.29^{* *}$ \\
& DSI & & & & -0.14 & $-0.33^{* *}$ & $-0.23^{*}$ & $0.28^{* *}$ \\
& DS & & & & & & $0.73^{* *}$ & $0.94^{* *}$ & $0.39^{* *}$ \\
& NSt (g) & & & & & & & $0.90^{* *}$ & $-0.26^{* *}$ \\
& DS & & & & & & & $0.12 \mathrm{~ns}$ \\
\hline
\end{tabular}

\section{Discussion}

The largest reduction in seed yield due to drought stress was in genotypes REDWOLAITA and ECAB 0412 confirming these genotypes were sensitive for drought stress and should not be recommended for drought stress areas in the region. Seed yield, pods per plant, 100-seed weight, and harvest index of small red seeded common bean genotypes were significantly affected by drought stress. The number of pods per plant, followed by seeds per pod were the most affected yield components under drought stress. This is consistent with reports on other legumes including common bean (Leport et al., 2006; Carlos et al., 2006; Setegn, 2006) that drought reduced biomass, seed yield, harvest index and seed weight of common bean. The same authors also reported that under the moderate drought; mean seed yield was reduced by $62 \%$. Moderate to severe drought stress reduced biomass and seed yield from $20 \%$ to $90 \%$, for harvest index, seeds per pod and days to maturity. Other researchers also reported reduction in biomass, number of seeds and pods per plant, days to maturity, harvest index, and seed yield in common bean by moderate to high drought stress (Ramirez-Vallejo \& Kelly, 1998; Acosta-Gallegos \& Adams, 1991). However, as suggested by many researchers the relative importance of individual components as determinants of seed yield varies from experiment to experiment (Ramirez-Vallejo \& Kelly, 1998; Shenkut \& Brick, 2003). From the present study genotypes which showed lowest values of PHI under drought stress conditions were not good to mobilize photosynthates to pod and seed. The genotypes with higher values of leaf area index, pod harvest index and lower values of pod wall biomass proportions showed with better yielding indicating greater ability to mobilize photosythates to production of pod and grain. In line with this result under field evaluation Polania et al. (2008) reported three drought adapted common bean genotypes and justified that the superior performance of these genotypes were associated with higher values of leaf area index, pod harvest 
index and lower values of pod wall biomass proportion. GM was considered for the superior index for evaluation of germplasm under DS and NS conditions (Schneider et al., 1997). Based on field performance during the experimental period at Goffa genotypes like ECAB-0427, ICTAJU-95-14, SER-16, SER-48, SER-125, SER-194 and RCB-592 with higher values of GM coupled with yield under stress condition may possibly be a good source of drought tolerant for genetic improvement.

Genotypes like SER-16 and ICTAJU-19-95-14 were also responsive in both under drought stress and non-stressed condition but SER-48 and SER-125 were well adapted under drought stress. Results of this study are similar to those reported by Schneider et al. (1997); Rosales-Serna et al. (2004) and Beebe et al. (2008) as there are some materials that were essentially selected for drought resistance also have better yield potential in favorable conditions but contrary to those predicted by Rosielle and Hamblin (1981). The later researchers suggested that high yielding genotypes in drought stress were likely to be low in yielding in non-stressed environment. Among the released varieties which were included in the study variety 'NASIER' with seed yield $908.2 \mathrm{~kg} / \mathrm{ha}$ performed relatively better than the others under drought condition and therefore it can be recommended for areas recurring soil moisture problem. The results obtained in this study are in agreement with the report of Singh (2001) that resistance to drought in modern common bean varieties might have been unintentionally reduced because of the emphasis of breeding have been mainly in introducing better resistance to biotic (insect, pest and diseases) rather than to abiotic stress (drought). The overall response to drought was as expected in both the resistance and susceptible genotypes. The selected drought resistance genotypes ECAB-0427, ICTAJU-95-14, SER-16, SER-48, SER-125, SER-194 and RCB-592 had the highest seed yield under DS while the drought susceptible genotypes like RED WOLIYTA and ECAB-0412 showed lower yield potential under DS. These results are consistent with previous reports (White et al., 1994; Teran \& Singh, 2002; Frahm et al., 2004). The reduction in pods per plant, number of seeds per pod and 100 seed weight due to drought contributed marked decrease in average grain yield (Acosta \& Kohashi, 1989; Acosta \& White, 1995; Barrios et al., 2005).

The presence of strong correlation between yields of drought stress and non-stress conditions indicate that genotypes which were performed under non-stress growing also performed under drought stress growing conditions. This result is also in agreements with the findings for Cattivelli et al. 2008, that explains traits maximizing productivity normally expressed in the absence of stress, can still sustain a significant yield improvement under mild to moderate stress. A high significant correlation $(\mathrm{r}=73)$ between yield in non-stress and drought stress conditions was also reported by (Rizza et al., 2004; quoted by Cattivelli et al., 2008) suggesting that, selection based on the absolute performance of the genotypes across environment is more successful than selecting across the minimum yield decrease under stress with respect to favorable condition. The negative correlation of seed yield under DS condition with percent reduction (PR) and drought susceptible index (DSI) were expected, since any genotypes with higher seed under drought stress growing conditions also have reduced values of percent reduction and drought susceptible index. Seed yield under drought were positively correlated with seed yield under non-stress environment $(\mathrm{r}=0.68)$ at drought stress level of $\mathrm{DII}=0.61$, because of a positive association between percent reduction (PR) and drought susceptibility index (DSI) for seed yield ( $\mathrm{r}=0.97)$, either trait could be used in combinations with the geometric mean yield (GM) to select drought resistant genotypes (Table 3). A positive correlation between seed yield in DS and NS environments supported similar findings by Ramirez-Vallejo and Kelly (1998) and Teran and Singh (2002).

\section{Conclusions and Recommendation}

Drought stress decreased seed yield and shoot biomass of the small red common bean genotypes in the post flowering period, this resulted in a difference between drought resistance and drought susceptible genotypes. Drought resistant genotypes maintained better biomass accumulation and partitioning to reproductive organs than the drought sensitive genotypes under drought stress conditions as it was correlated to seed yield. Generally, there were differences found in seed yield, biomass accumulation and allocations among bean genotypes tested under drought conditions and those genotypes with efficient biomass partitioning to reproductive structures were better adapted to drought stress. Thus strong partitioning of shoot biomass to reproductive structures contributed to increase seed in yield in common beans. Out of the released varieties which were included in the study "NASIER" performed relatively better than others released varieties under drought condition and therefore it can be recommended for areas like Goffa where recurring soil moisture problem. In addition to yield components and plant attributes, the efficiency of partitioning of photosynthates to grain yield has also been the reliable approach to identify drought resistant genotypes under moisture stress conditions. Based on the result of geometric mean (GM), drought susceptible index (DSI) and percent yield reduction (PR) genotypes were identified with greater yield potential under the tested environment. In common beans, better understanding of 
the key adaptive morphological and physiological traits and mechanisms that are associated with growth, biomass partitioning and yield under drought stress conditions can contribute to development of rapid and reliable selection criteria that are needed to identify drought resistant genotypes.

\section{Acknowledgments}

We appreciate the assistance of Melkassa (MARC) and Awassa (AARC) Agricultural Research Centers in germplasm introduction and collection. We also acknowledge Southern Agricultural Research Institute (SARI) for the financial support during the experiment.

\section{References}

Abebe, A., Brick, M. A., \& Kirkby, R. (1998). Comparison of selection indices to identify productive common bean lines under diverse environmental conditions. Field Crops Res., 58, 15-23. http://dx.doi.org/10.1016/S0378-4290(98)00082-3

Acosta-Gallegos, J. A ., \& Adams, M. W. (1991). Plant traits and yield stability of common bean (Phaseolus vulgaris L.) cultivars under drought stress. J. Agric. Sci., 117, 213-219. http://dx.doi.org/10.1017/S0021859600065308

Acosta-Gallegos, J. A. (1988). Selection of common bean (Phaseolus vulgaris L.) genotypes with enhanced drought tolerance and biological nitrogen fixation. Ph D diss Michgan state University East Lansing (Diss Abstr 88-24816).

Acosta-Gallegos, J. A., \& Kohashi-Shibata, J. (1989). Effect of water stress on growth and yield of indeterminate dry bean (Phaesolus vulgaris) cultivars. Field Crop Res., 20, 81-90. http://dx.doi.org/10.1016/0378-4290(89)90054-3

Acosta-Gallegos, J. A., \& White, J. W. (1995). Phenological plasticity as an adaptation by common bean to $\begin{array}{lllll}\text { rainfed } & \text { environments. } & \text { Crop } & \text { Sci., } & 35,\end{array}$ http://dx.doi.org/10.2135/cropsci1995.0011183X003500010037x

Asrat, A., Daniel, D., \& Yasin, G. (2006). Breeding Legumes for increased yield and stress resistance in southern Ethiopia. pp. 105-111. In: Food and Forage Legumes of Ethiopia: Progress and Prospects. Proceedings of the workshop on food and forage legumes, Addis Ababa, Ethiopia.

Barrios, A., Hoogenboom, G., \& Nesmith, D. S. (2005). Drought stress and the distribution of vegetative and reproductive traits of a bean cultivar. Sci.agric. (Piracicaba, Braz.), 62(1), 18-22.

Beebe, S. I., Rao, M., \& Butare, L. (2008). Breeding for abiotic stress tolerance in common bean: present and future challenges. Proceedings of the 14th Australian Plant Breeding \& 11th SABRAO Conference, 10 to 14 August, 2009, Brisbane, Australia (invited paper).

Broughton, W. J. G., Hernandez, M., Blair, S., Beebe, P., \& Vanderleyden, J. (2003). Bean (Phaseolus spp.)model food legumes. Plant Soil, 252, 55-128. http://dx.doi.org/10.1023/A:1024146710611

Blum, A., \& Ebercon, A. (1981). Cell membrane stability as a measure of drought and heat tolerance in wheat. Crop Science, 21, 43-47. http://dx.doi.org/10.2135/cropsci1981.0011183X002100010013x

Cattivelli, L., Rizza, F., Badeck, F. W. E., Muzzucotelli, A. M., Mestrangelo, E., Francia, C., ... Stanca, A. M. (2008). Drought tolerance improvement in crop plants: An integrated view from breeding to genomics. Field Crop Res., 105, 1-4. http://dx.doi.org/10.1016/j.fcr.2007.07.004

Centro Internacional de Agricultura Tropical (CIAT). (1995). Catalog of advanced bean lines from CIAT. (2nd ed.). Macro Antonio Rodriguiz, Hector Fabio Rairez, Maria Clara Valencia, Oswaldo V.Voysest, and Jeffrey W. White (comps.). CIAT, Cali, Colombia. p. 298

Chaves, M. M., Pereira, J. S., Maroco, J. P., Rodrigues, M. L., Ricardo, C. P. P., Osorio, M. L., ...Pinheiro, C. (2002). How plants cope with water stress in the field: photosynthesis and growth. Ann Bot., 89, 907-916. http://dx.doi.org/10.1093/aob/mcf105

Fernandez, G. C. J. (1993). Effective selection criteria for assessing plant stress tolerance. In: Adaptation of food crops to temperature and water stress, C.G. Kuo (ed.). AVRDC, Shanhua, Taiwan, pp. 257-270.

Fischer, R. A., \& Maurer, R. (1978). Drought resistance in spring wheat cultivars. I. Grain yield responses. Aust. J. Agric. Res., 29, 897-912. http://dx.doi.org/10.1071/AR9780897

Frahm, M. A., Rosas, J. C., Mayek-Pérez, N., López-Salinas, E., Acosta-Gallegos, J. A., \& Kelly, J. D. (2004). Breeding beans for resistance to terminal drought in the lowland tropics. Euphytica, 136, 223-232. 
http://dx.doi.org/10.1023/B:euph.0000030671.03694.bb

Gomez, A. K., \& Gomez, A. A. (1984). Statistical Procedures for Agricultural Research. (2nd ed.). New York: John Wiley and Sons.

Leport, L., Turner, N. C., Davies, S. L., \& Siddique, K. H. M. (2006). Variation in pod production and abortion among chickpea cultivars under terminal drought. European Journal of Agronomy, 24(3), $236-246$. http://dx.doi.org/10.1016/j.eja.2005.08.005

Muñoz-Perea, C. G., Terán, H., Allen, R. G., Wright, J. L., Westermann, D. T., \& Singh, S. P. (2006). Selection for Drought Resistance in Dry Bean Landraces and Cultivars. Crop Sci. Soc. of America, 46, 2111-2120.

Polania, A., Polania, J., Grajales, M., Cajiao, C., Garacia, R., Ricaurte, J., ... Rao, I. M. (2008). Physiological evaluation of drought resistance in elite lines of common bean (Phaseolus vulgaries L.) under field conditions. CIAT, A.A 6713, Cali, Colombia.

Ramirez-Vallejo, P., \& Kelly, J. D. (1998). Traits related to drought resistance in common bean. Euphytica, 99, 127-136. http://dx.doi.org/10.1023/A:1018353200015

Rao, I. M., Beebe, S. E., Polanía, J., Grajales, M., Cajiao, C., García, R., ... Rivera, M. (2009). Physiological basis of improved drought resistance in common bean: the contribution of photosynthate mobilization to grain. Paper presented at Inter drought III: The 3rd International Conference on Integrated Approaches to Improve Crop Production under Drought-Prone Environments, October 11-16, 2009, Shanghai, China.

Rosales-Serna, R., Kohashi-Shibata, J., Acosta-Gallegos, J. A., Trejo-Lopez, C., Ortiz- Cereceres, J., \& Kelly, J. D. (2004). Biomass distribution, maturity acceleration and yield in drought stressed common bean cultivars. Field Crops Res., 85, 203-211. http://dx.doi.org/10.1016/S0378-4290(03)00161-8

Rosielle, A. A., \& Hamblin, J. (1981). Theoretical aspects for yield in stress and non-stress environments. Crop Sci., 21, 943-946. http://dx.doi.org/10.2135/cropsci1981.0011183X002100060033x

Setegn Gebeyehu. (2006). Physiological Responses to Drought Stress of common bean (Pharsalus vulgaries L) Genotypes Differing in Drought Resistance. Doctorial Dissertation submitted to Justus-Liebig University of Giessen, Germany.

Schneider, K. A., Brothers, M. E., Kelly, J. D. (1997). Marker-assisted selection to improve drought resistance in common bean. Crop Sci., 37, 37-51. http://dx.doi.org/10.2135/cropsci1997.0011183X003700010008x

Shenkut, A. A., \& Brick, M. A. (2003). Traits associated with dry edible bean (Phaseolus vulgaris L.) productivity under diverse soil moisture environments. Euphytica., 133(3), 339-347. http://dx.doi.org/10.1023/A:1025774110004

Siddique, K. H. M., Regan, L. K., Tennant, D., \& Thompson, B. D. (2001). Water use and water use efficiency of cool season grain legumes in low rainfall Mediterranean type environments. Eur. J. Agron., 15, $267-280$. http://dx.doi.org/10.1016/S1161-0301(01)00106-X

Singh, S. P. (2001). Broadening the genetic base of common bean cultivars: a review. Crop Sci., 41, 1659-1675. http://dx.doi.org/10.2135/cropsci2001.1659

Teran, H., \& Singh, S. P. (2002). Comparison of sources and lines selected for drought resistance in common bean. Crop Sci., 42, 64-70. http://dx.doi.org/10.2135/cropsci2002.0064

Visser, B. (1994). Technical aspects of drought tolerance. Biotechnology and Development Monitor, No. 18 , p. 5.

White, J. W., Ochoa, R., Ibarra, F., \& Singh, S. P. (1994). Inheritance of seed yield, maturity and seed weight of common bean (Phaseolus vulgaris L.) under semi-arid rain fed conditions. J Agric Sci, 122, $265-273$. http://dx.doi.org/10.1017/S0021859600087451

Wortmann, C. S, Kirby, R. A., Eledu, C. A., \& Allen, D. J. (1998). Atlas of common bean Phaseolus vulgaris L. production in Africa. CIAT Pan-African Bean Research Alliance. 